\title{
The Effect of Endoscopic Tympanoplasty on Cochlear Function
}

\author{
Isa Kaya ${ }^{1} \cdot$ Goksel Turhal $^{2} \cdot$ Arin Ozturk $^{1} \cdot$ Sercan Gode ${ }^{1} \cdot$ Cem Bilgen $^{1} \cdot$ Tayfun Kirazli ${ }^{1}$ \\ ${ }^{l}$ Department of Otolaryngology, Ege University School of Medicine, Izmir; ${ }^{2}$ Department of Otolaryngology, Selcuk State Hospital, Izmir, Turkey
}

Objectives. The aim of this prospective clinical study was to measure the audiologic outcomes of the patients that underwent endoscopic transcanal cartilage tympanoplasty, and to investigate the effects on cochlear function.

Methods. Thirty-three patients (33 ears) who were diagnosed with noncomplicated chronic otitis media and underwent endoscopic transcanal cartilage tympanoplasty technique were included. Pre- and postoperative first month distortion product otoacoustic emission (DPOAE) signal-to-noise ratio (SNR), bone conduction hearing levels and air bone gap (ABG) values were measured and total endoscope usage time was noted.

Results. Preoperative median SNR measurements at $0.5,1,2$, and $4 \mathrm{kHz}$ were $6 \mathrm{~dB}$ (1-11; interquartile range [IQR], 1$), 6$ $\mathrm{dB}$ (4-20; IQR, 1), $7 \mathrm{~dB}$ (3-26; IQR, 5) and $5.50 \mathrm{~dB}$ (0-9; IQR, 3), respectively. Postoperative median SNR measurements at $0.5,1,2$, and $4 \mathrm{kHz}$ were $6 \mathrm{~dB}(3-9$; IQR, 1), $6 \mathrm{~dB}$ (2-21; IQR, 3), $7 \mathrm{~dB}(2-20$; IQR, 3), and $6 \mathrm{~dB}(0-10$; IQR, 7), respectively. Regarding the DPOAE measurements, there was no statistically significant difference between the SNR values of all given frequencies $(P>0.05)$. Regarding the pure tone audiometry (PTA) measurements, bone conduction was significantly better at 0.5 and $1 \mathrm{kHz}$, postoperatively $(P<0.05)$ and there was statistically significant difference at 2 and $4 \mathrm{kHz}(P>0.05)$. Additionally, no statistically significant correlation was found between the SNR and PTA measurements and the endoscope usage time $(P>0.05)$.

Conclusion. We suggested that cochlear functions and sensorineural hearing remained stable after endoscopic transcanal cartilage tympanoplasty and cold light source doesn't cause significant adverse effects cochlear functions.

Keywords. Endoscopic Ear Surgery; Endoscopic Tympanoplasty; Cold Light Source; Otoacoustic Emission

\section{INTRODUCTION}

In the last two decades, endoscopes have been frequently used in otological surgery. First, they were only used for diagnostic procedures but soon after they became the standard of care especially in sinus surgery. These instruments had gained a widespread use in many otologic procedures such as tympanoplasty. Tympanoplasty is performed to repair the perforated tympanic membrane (TM) and rehabilitate the hearing in patients with

- Received April 23, 2017

Revised August 30, 2017

Accepted October 27, 2017

- Corresponding author: Arin Ozturk

Department of Otolaryngology, Ege University School of Medicine, 35100,

Bornova, Izmir,Turkey

Tel: +90-232-390-2600, Fax: +90-232-390-2670

E-mail: arinozturk@yahoo.com chronic otitis media [1]. Surgeons can perform this procedure transcanal with endoscopes and this is the main reason that makes endoscopic transcanal tympanoplasty technique popular. The pros and cons of using these instruments in otologic surgery have been questioned frequently. Many animal and ex vivo studies investigated the effect of temperature changes caused by rigid endoscopes. In an experimental animal study conducted on guinea pigs, Dundar et al. [2] demonstrated that the temperature increase in the oval window occurred because of the reflected light transmitted to the perilymph. The authors of this study suggest that all light sources could cause a temperature rise in inner ear fluids. The physical effect of the thermal injury is known however functional changes in the human cochlea remain unclear. The aim of this study was to measure the audiologic outcomes of the patients that underwent endoscopic transcanal cartilage tympanoplasty, and to investigate the effect of

Copyright $@ 2018$ by Korean Society of Otorhinolaryngology-Head and Neck Surgery.

This is an open-access article distributed under the terms of the Creative Commons Attribution Non-Commercial License (http://creativecommons.org/licenses/by-nc/4.0)

which permits unrestricted non-commercial use, distribution, and reproduction in any medium, provided the original work is properly cited. 
endoscopic transcanal tympanoplasty on cochlear function.

\section{MATERIALS AND METHODS}

This study was performed at the Department of Otolaryngology of Ege University School of Medicine between January 2016 and June 2016. All procedures performed in studies involving human participants were in accordance with the ethical standards of the institutional and/or national research committee and with the 1964 Helsinki Declaration and its later amendments or comparable ethical standards. The study was approved by the Institutional Review Board of Ege University Hospital (IRB No. 176/16). Informed consent was obtained from all individual participants included in the study.

\section{Patient selection}

The study was designed as a prospective study. All of the data were prospectively collected. These included demographic data, date of the surgery, total time period of the endoscope usage, pre- and postoperative pure tone audiometry (PTA), pre- and postoperative distortion product otoacoustic emission (DPOAE) measurements.

Thirty-three patients (33 ears) who had TM perforation with noncomplicated chronic otitis media were included. All of the patients were operated with the endoscopic transcanal cartilage tympanoplasty technique. Selection criteria for the study were: perforation with a history of at least 1 year, presence of intact ossicular chain, absence of clinically or radiologically demonstrated cholesteatoma, no evidence of inflammation or discharge at the time of surgery, well healed cartilage graft presence with no effusion in middle ear at postoperative first month examination.

\section{Instrumentation and procedure}

A high definition monitor and camera (Karl Storz, Tuttlingen, Germany) were used, and videos were recorded on a computer hard disc. Xenon light source (Xenon Nova 175, Karl Storz), and $4 \mathrm{~mm}$ (18 cm length) Hopkins-rod lens rigid $0^{\circ}$ endoscope

\section{H I G G H L I G G H T S}

- Pre- and postoperative first month distortion product otoacoustic emission (DPOAE) signal-to-noise ratio, bone conduction hearing levels and air bone gap values of patients that underwent endoscopic transcanal cartilage tympanoplasty were measured.

- There was no significant decrease in DPAOE measurements at all frequencies.

- Total endoscope usage time around 25 to 30 minutes does not have a negative effect on cochlear functions.

- Cochlear functions and sensorineural hearing remained stable after endoscopic transcanal cartilage tympanoplasty.
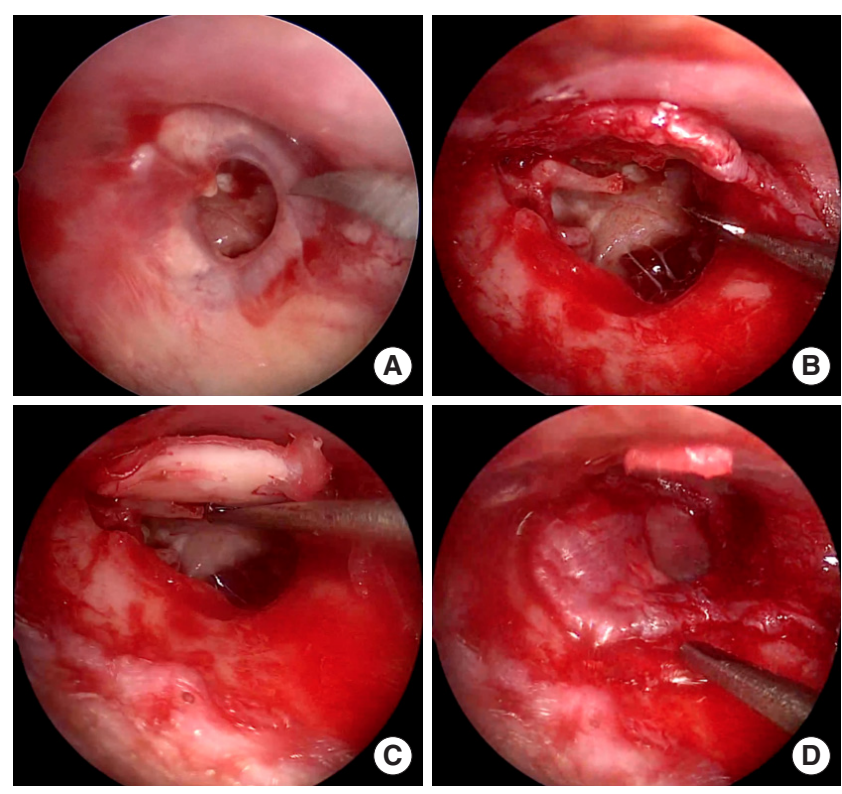

Fig. 1. (A) Central tympanic membrane perforation, (B) tympanomeatal flap incision and anteriorly based tympanomeatal flap elevation, (C) cartilage graft placing, (D) closure of tympanic membrane perforation.

(Karl Storz) were used during the procedures. All patients were operated by the same senior surgeon (IK) under general anaesthesia.Tragal-perichondrial island graft was used for grafting and the cartilage graft was thinned to $0.2-0.5 \mathrm{~mm}$. Margins of the TM were freshened. An anteriorly based tympanomeatal flap and the annulus were raised. The graft was placed in using the over-underlay technique, lateral to the handle of malleus and medial to the TM and annulus. The external auditory canal was packed with antibiotic ointment. The followings are precautions that were taken by surgeon: (1) submaximal use of the endoscopes (maximum 1 minute in external auditory canal at one pass); (2) endoscope's tip was hold laterally to TM, and never placed in middle ear cavity; (3) middle ear cavity and endoscope's tip is periodically irrigated with saline solution $\left(26^{\circ} \mathrm{C}\right)$ every 2 minutes. Total endoscope usage was noted (Fig. 1).

PTA and DPOAE were measured before the surgery and at postoperative first month control. Standard PTA (Interacoustics AC40, Middelfart, Denmark; headphone: TDH39) was performed before the surgery and pure tone bone conduction audiometries for the frequencies of 500,1,000, 2,000, and 4,000 Hz. The GSI 60 DPOAE system (MED-EL, Innsbruck, Austria) was used for DPOAE measurements. Otoacoustic emissions were measured using stimuli in different frequencies and intensities. Stimulus intensities were L1:65 dB sound pressure level (SPL) and L2:55 dB SPL. The two different frequencies (f1 and f2) were set as $\mathrm{f} 2 / \mathrm{f} 1=1.22$. Measurements were performed at 500, 1,000, 2,000, and 4,000 $\mathrm{Hz}$ frequencies. The differences between the distortion product emission and the noise floor amplitudes were calculated to evaluate signal-to-noise ratio (SNR) and responses. 


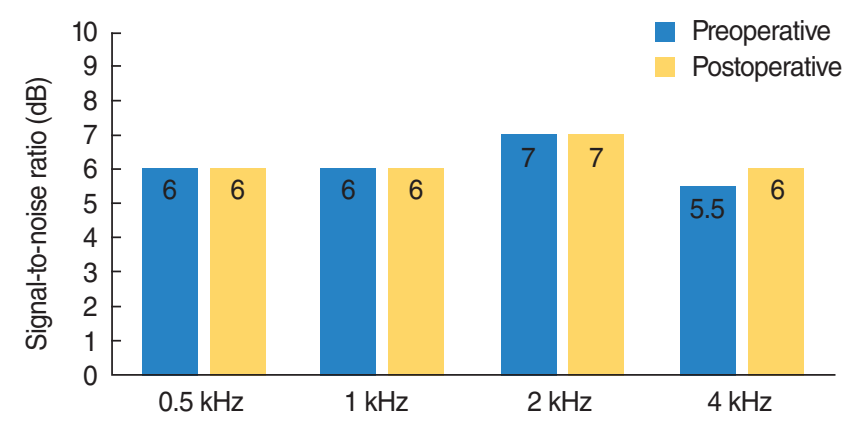

Fig. 2. Pre- and postoperative signal-to-noise ratio measurements.

\section{Outcomes}

The effect of endoscopy and light source on cochlear function was assessed by pre- and postoperative SNR and bone conduction hearing level (HL) measurements. Additionally, the relation between the endoscope usage duration and the cochlear function was evaluated.

\section{Statistical analysis}

Statistical analysis was made using software IBM SPSS ver. 22.0 (IBM Corp., Armonk, NY, USA). Chi-square exact tests were used for the comparison of categorical data. Independent and paired sample $t$-tests were used for the analysis of parametric variables while Wilcoxon and Mann-Whitney $U$-tests were used for the analysis of nonparametric variables based on the distribution pattern of the data. The Shapiro-Wilk test was used for determining the distribution pattern of the data. Correlation analysis was performed via Pearson or Spearman correlation analysis based on the distribution pattern of the data. The distribution of the groups of SNR measurements was nonparametric. Data were expressed as median and interquartile range (IQR). On PTA measurements, distribution of the groups of HL measurements was parametric. Data were expressed as mean \pm standard deviation. A $P$-value less than 0.05 was considered statistically significant.

\section{RESULTS}

The 33 patients included in the study had a mean age of $36.9 \pm$ 11.9 years (range, 17 to 60 years; 23 females and 10 males). Mean endoscope usage time was $27.2 \pm 3.6$ minutes and mean surgery time was $36.8 \pm 5.9$ minutes. There was subtotal perforation that larger than $\% 50$ of TM in seven patients. Preoperative median SNR measurements at $0.5,1,2$, and $4 \mathrm{kHz}$ were $6 \mathrm{~dB}$ (1-11; IQR, 1), $6 \mathrm{~dB}$ (4-20; IQR, 1), $7 \mathrm{~dB}$ (3-26; IQR, 5) and $5.50 \mathrm{~dB}(0-9 ; \mathrm{IQR}, 3)$, respectively. Postoperative median SNR measurements at $0.5,1,2$, and $4 \mathrm{kHz}$ were $6 \mathrm{~dB}(3-9 ; \mathrm{IQR}, 1)$, $6 \mathrm{~dB}$ (2-21; IQR, 3), $7 \mathrm{~dB}$ (2-20; IQR, 3), and $6 \mathrm{~dB}$ (0-10; IQR, $7)$, respectively. Regarding the DPOAE measurements, there was no statistically significant difference between the SNR val-

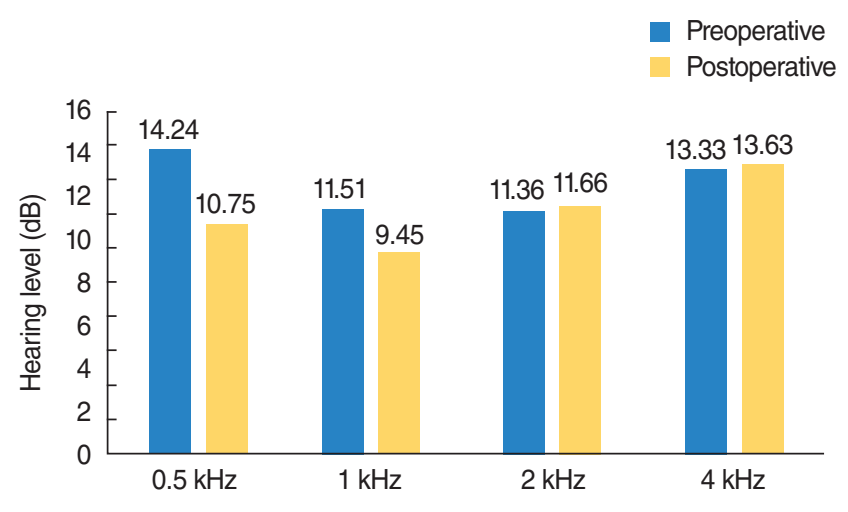

Fig. 3. Pre- and postoperative pure tone bone conduction audiometry levels ( $\mathrm{dB}$ hearing level).

ues for all given frequencies, also in subtotal perforation group too $(P>0.05)$ (Fig. 2$)$.

Preoperative mean pure tone bone conduction audiometry levels at $0.5,1,2$, and $4 \mathrm{kHz}$ were $14.24 \pm 9.44 \mathrm{~dB} \mathrm{HL}, 11.51 \pm$ $7.85 \mathrm{~dB}$ HL, $11.36 \pm 9.32 \mathrm{~dB} \mathrm{HL}$, and $13.33 \pm 9.37 \mathrm{~dB}$ HL, respectively. Postoperative mean pure tone bone conduction audiometry levels at $0.5,1,2$, and $4 \mathrm{kHz}$ were $10.75 \pm 8.30 \mathrm{~dB} \mathrm{HL}$, $9.45 \pm 7.64 \mathrm{~dB}$ HL, $11.66 \pm 9.07 \mathrm{~dB}$ HL, and $13.63 \pm 10.98 \mathrm{~dB}$ HL, respectively (Fig. 3). Mean air bone gap (ABG) improvement (dB SPL) at $0.5,1,2$, and $4 \mathrm{kHz}$ were $12.03 \pm 7.16 \mathrm{~dB} \mathrm{HL}$, 9.06 $\pm 7.77 \mathrm{~dB} \mathrm{HL}, 9.84 \pm 7.67 \mathrm{~dB} \mathrm{HL}$, and $6.56 \pm 9.28 \mathrm{~dB} \mathrm{HL}$, respectively. Regarding the PTA measurements, bone conduction was significantly better at 0.5 and $1 \mathrm{kHz}$ postoperatively $(P<0.05)$ and there was not statistically significant difference at 2 and $4 \mathrm{kHz}(P>0.05)$. Postoperative ABG improvements were statistically significant at all given frequencies $(P<0.05)$. Additionally, no statistically significant correlation was found between the SNR and PTA measurements and the endoscope usage time $(P>0.05)$.

\section{DISCUSSION}

Comparing the transcanal approach with the classical retroauricular and endaural approach, it has the advantage of being less invasive. Endoscopes can provide wider and more detailed visualization from different angles. Hence, surgeons get chance to work effectively in external auditory canal and middle ear without canaloplasty or a postauricular incision. Besides using endoscopes in this procedure enables having a total exposure of TM [1]. Thanks to modern technology, endoscopes have smaller diameter now, and video recorders can provide images in high definition. All these instruments are now better optimized for endoscopic ear surgery.

During endoscopic otologic surgery, the temperature change during endoscope use has been questioning whether if this increase of temperature might be harmful for inner ear structures. 
A study that aims measuring the thermal properties of operative endoscopes used in otorhinolaryngological practice, showed that the tip of rigid endoscopes could cause tissue damage with the temperature rise [3]. Another study concluded that otoendoscopes used in otologic surgery could increase the temperature in the lateral semicircular canal and cause vertigo [4].

The neurosensorial cells of the cochlea are highly sensitive to environmental factors such as noise, trauma and changes in temperature and outer hair cells are the first cells to be affected [5]. Stimulated outer hair cells evoke the otoacoustic emissions and otoacoustic emission testing gives results that are objective, frequency-specific, reliable, and stable [6]. Additionally, the test is short and requires minimal patient cooperation. Thus, DPOAE was performed to measure cochlear functions. It was assumed that using a cartilage graft and effusion in middle ear cavity could decrease DPOAE. So, the cartilage graft was thinned and the effusion was excluded with both tympanometry and clinical examination. Regarding the PTA measurements, postoperative neurosensorial hearing was better at 0.5 and $1 \mathrm{kHz}$ and there was no statistically significant difference at 2 and $4 \mathrm{kHz}$ frequencies. The reason of lower ABG levels and better bone-conduction HLs at 0.5 and $1 \mathrm{kHz}$ measured with PTA is thought to be related the patients' subjective better neurosensorial hearing responses. There was no significant decrease in DPAOE measurements at all frequencies. Hence, it is suggested that cold light source does not cause permanent changes in cochlear functions. Additionally, it is thought that a total endoscope usage time around 25 to 30 minutes does not have a negative effect on cochlear functions.

Aksoy et al. [7] performed an experimental animal study on guinea pigs to investigate the thermal effects of cold light sources and endoscopes on the inner ear. They found that the thermal effect generated by an endoscope with a Xenon cold light source could decrease DPOAE values and increase auditory brainstem response thresholds. They suggested that, to avoid tissue injury due to increased heat at the tip of the endoscope, middle ear should not be exposed to the endoscope for a long-time period, periodical cold irrigation should be applied to the endoscope and LED (light-emitting diode) light source with a $2.7 \mathrm{~mm}$ diameter endoscope should be preferred. In our study, a Xenon cold light source and 4-mm diameter rigid $0^{\circ}$ endoscope was used. According to our results, if the surgeon pays attention to submaximal usage of endoscopes and periodical irrigation of the middle ear cavity and endoscope's tip, it is unlikely to affect the cochlear functions adversely with thermal trauma cause by endoscopes and light source even with a $4 \mathrm{~mm}$ endoscope and a Xenon light source. This study was performed on tympanoplasty patients and mean endoscope usage times were relatively short when compared other surgeries. Besides that, patient number of our study creates limitation in statistical analysis of audiologic outcomes. Future studies to investigate the impact of longer operative times and different light sources or endoscopes are need- ed to fully assess the impact on cochlear function.

One-handed instrumentation is seen as a surgical handicap during endoscopic ear surgery. Surgeons lose the chance to perform dissection and aspiration manoeuvres at the same time [8]. To avoid that endoscope holders could be used. A study on fresh human temporal bones aimed measuring temperature changes during rigid middle ear endoscopy in a human temporal bone model and investigate whether suction can act as a significant cooling mechanism [9]. According to their findings, Xenon light sources could cause a temperature rise up to $46.9^{\circ} \mathrm{C}$ and they recommended the submaximal use of endoscopes and suctioning of the middle ear. Similarly, in this study it is suggested that taking out the endoscope from external auditory canal and cooling it with irrigation plays an important role to avoid thermal damage. Khan and Parab [10] described an endoscope holder-assisted transcanal two-handed endoscopic tympanoplasty procedure with better surgical outcomes and they suggested that some simple techniques like irrigation should be used during surgery to prevent thermal damage. According to the findings of this study if the surgeon pays attention to aforementioned precautions, these instruments could be safely used in otologic procedures with short duration.

In conclusion, cochlear functions and sensorineural hearing remained stable after endoscopic transcanal cartilage tympanoplasty. This study is the first study to reveal the thermal effects of endoscope use on human cochlea and hearing in endoscopic tympanoplasty patients. In order to better understand the thermal effects of the light sources on human cochlea, randomized clinical studies with longer otologic procedures, different light sources and endoscope types are warranted.

\section{CONFLICT OF INTEREST}

No potential conflict of interest relevant to this article was reported.

\section{ACKNOWLEDGMENTS}

This study was presented as a poster presentation at the 38th Turkish National Congress of Otorhinolaryngology-Head and Neck Surgery on 26-30 October, 2016 in Antalya,Turkey.

\section{REFERENCES}

1. Ayache S. Cartilaginous myringoplasty: the endoscopic transcanal procedure. Eur Arch Otorhinolaryngol. 2013 Mar;270(3):853-60.

2. Dundar R, Bulut H, Yukkaldiran A, Guler OK, DemirtasY, Iynen I, et al. Temperature rises in the round window caused by various light sources during insertion of rigid endoscopes: an experimental animal study. Clin Otolaryngol. 2016 Feb;41(1):44-50. 
3. MacKeith SA, Frampton S, Pothier DD.Thermal properties of operative endoscopes used in otorhinolaryngology. J Laryngol Otol. 2008 Jul;122(7):711-4.

4. Bottrill I, Perrault DF Jr, Poe D. In vitro and in vivo determination of the thermal effect of middle ear endoscopy. Laryngoscope. 1996 Feb;106(2 Pt 1):213-6.

5. Lonsbury-Martin BL, Martin GK. Noise-induced hearing loss. In: Flint PW, Haughey BH, Lund VJ, Niparko JK, Robbins KT, Thomas JR, et al, editor. Cummings otolaryngology: head and neck surgery. 6th ed. Philadelphia (PA): Saunders Elsevier; 2014. p. 2345-58.

6. Lonsbury-Martin BL, Martin GK. The clinical utility of distortionproduct otoacoustic emissions. Ear Hear. 1990 Apr;11(2):144-54.

7. Aksoy F, Dogan R, Ozturan O, Eren SB, Veyseller B, Gedik O. Ther- mal effects of cold light sources used in otologic surgery. Eur Arch Otorhinolaryngol. 2015 Oct;272(10):2679-87.

8. Badr-El-Dine M, James AL, Panetti G, Marchioni D, Presutti L, Nogueira JF. Instrumentation and technologies in endoscopic ear surgery. Otolaryngol Clin North Am. 2013 Apr;46(2):211-25.

9. Kozin ED, Lehmann A, Carter M, Hight E, Cohen M, Nakajima HH, et al.Thermal effects of endoscopy in a human temporal bone model: implications for endoscopic ear surgery. Laryngoscope. 2014 Aug; 124(8):E332-9.

10. Khan MM, Parab SR. Endoscopic cartilage tympanoplasty: a twohanded technique using an endoscope holder. Laryngoscope. 2016 Aug;126(8):1893-8. 